\title{
Skeletal muscle molecular responses to resistance training and dietary supplementation in COPD
}

\author{
Despina Constantin, ${ }^{1}$ Manoj K Menon, ${ }^{2}$ Linzy Houchen-Wolloff, ${ }^{2}$ Michael D Morgan, ${ }^{2}$ \\ Sally J Singh, ${ }^{2}$ Paul Greenhaff, ${ }^{1}$ Michael C Steiner ${ }^{2}$
}

\begin{abstract}
- Additional material is published online only. To view please visit the journal online (http://dx.doi.org/10.1136/ thoraxjn-2012-202764)

${ }^{1}$ MRC/ARUK Centre for Musculoskeletal Ageing Research, School of Biomedical Sciences, University of Nottingham, Nottingham, UK ${ }^{2}$ Department of Respiratory Medicine, Institute for Lung Health, University Hospitals of Leicester NHS Trust, Glenfield Hospital, Leicester, UK
\end{abstract}

Correspondence to Dr Michael C Steiner, Department of Respiratory Medicine, Institute for Lung Health, University Hospitals of Leicester NHS Trust, Glenfield Hospital, Groby Road Leicester LE3 9QP, UK : michael.steiner@uhl-tr.nhs.uk

DC and MKM are joint first authors. PG and MCS are joint senior authors.

Received 22 September 2012 Revised 23 January 2013 Accepted 7 March 2013 Published Online First 27 March 2013
To cite: Constantin $D_{\text {, }}$ Menon MK, HouchenWolloff $\mathrm{L}$, et al. Thorax 2013;68:625-633

\begin{abstract}
Background Skeletal muscle dysfunction is a systemic feature of chronic obstructive pulmonary disease (COPD), contributing to morbidity and mortality. Physical training improves muscle mass and function in COPD, but the molecular regulation therein is poorly understood. Methods Candidate genes and proteins regulating muscle protein breakdown (ubiquitin proteasome pathway), muscle protein synthesis (phosphatidylinositol 3 kinase/Akt/mammalian target of rapamycin pathway), myogenesis (MyoD, myogenin and myostatin) and transcription (FOXO1, FOXO3 and RUNX1) were determined in quadriceps muscle samples taken at four time points over 8 weeks of knee extensor resistance training (RT) in patients with COPD and healthy controls (HCs). Patients with COPD were randomly allocated to receive protein/carbohydrate or placebo supplements during RT.
\end{abstract}

Results 59 patients with COPD (mean (SD) age 68.0 (9.3) years, forced expiratory volume in $1 \mathrm{~s}\left(\mathrm{FEV}_{1}\right) 46.9$ (17.8) \% predicted) and $21 \mathrm{HCs}$ (66.1 (4.8) years, 105.0 (21.6) \% predicted) were enrolled. RT increased lean mass $(\sim 5 \%)$ and strength $(\sim 20 \%)$ in all groups. Absolute work done during RT was lower throughout in patients with COPD compared with HCs. RT resulted in increases (from basal) in catabolic, anabolic, myogenic and transcription factor protein expression at $24 \mathrm{~h}$, 4 weeks and 8 weeks of exercise in HCs. This response was blunted in patients with COPD, except for myogenic signalling, which was similar. Nutritional

supplementation did not augment functional or

molecular responses to RT.

Conclusions The potential for muscle rehabilitation in response to RT is preserved in COPD. Except for markers of myogenesis, molecular responses to RT are not tightly coupled to lean mass gains but reflect the lower work done during RT, suggesting some caution when identifying molecular targets for intervention. Increasing post-exercise protein and carbohydrate intake is not a prerequisite for a normal training response in COPD.

\section{INTRODUCTION}

Reduced skeletal muscle mass and function is an important clinical feature of chronic obstructive pulmonary disease (COPD) which compromises physical performance $\mathrm{e}^{1-3}$ and predicts morbidity and mortality independently from lung function impairment. ${ }^{4-7}$ This is important because muscle is a potential therapeutic target in a disease in which the primary lung pathology is frequently irreversible. ${ }^{8}$ Proof that this approach is beneficial is demonstrated by the efficacy of physical training in

\section{Key messages}

What is the key question?

- Impaired skeletal muscle function is a potentially remediable systemic manifestation of chronic obstructive pulmonary disease (COPD). Resistance exercise training improves muscle mass and function in patients with COPD, but molecular mechanisms underpinning these adaptations are poorly understood and the impact of post-exercise dietary supplementation aimed at increasing muscle protein accretion has not been investigated.

What is the bottom line?

- We show that thigh lean mass and strength gains with training are similar in patients with COPD and healthy controls. With the exception of myogenic proteins, molecular responses to training are uncoupled from these functional gains and more closely reflect absolute workloads performed during training. Post-exercise protein/carbohydrate supplementation does not augment molecular or functional responses.

\section{Why read on?}

- We report a detailed time-course investigation in COPD of the responses of an unprecedented range of candidate genes and proteins known to regulate muscle protein synthesis, breakdown and myogenesis to resistance training and nutritional supplementation. The findings have implications for identification of drug targets aimed at improving muscle function in COPD.

improving muscle function in COPD. ${ }^{9}{ }^{10}$ However, the molecular and cellular events underpinning muscle dysfunction and its response to training in COPD are poorly understood and it remains unclear whether the potential for training-induced muscle adaptation is preserved in COPD compared with similar aged healthy subjects.

Cross-sectional comparative studies of patients with COPD and aged-matched healthy subjects have proposed muscle protein breakdown (MPB) as a driver of wasting in COPD. ${ }^{11-13}$ However, in the absence of data depicting temporal changes in 
muscle protein turnover or the expression of genes and proteins thought to regulate muscle mass in response to intervention, firm conclusions on the mechanistic and therefore clinical relevance of $\mathrm{MPB}$ to wasting in COPD cannot be drawn. Importantly, training-induced molecular adaptations will precede muscle mass gains and therefore may be missed if analysis is restricted to biopsy samples obtained when training is completed.

Resistance training (RT) alone or combined with post-exercise dietary protein supplementation increases muscle protein synthesis (MPS) via activation of mammalian target of rapamycin (mTOR) signalling. ${ }^{14-16}$ There is evidence that MPS via this signalling pathway and insulin-mediated inhibition of MPB are blunted in older volunteers, ${ }^{17-20}$ and that the timing of postexercise supplementation may be important to the magnitude of the anabolic response in older people. ${ }^{21}$ The role of protein supplementation combined with RT in patients with COPD has not been elucidated.

We conducted a detailed investigation of genes and proteins, thought to play a role in muscle mass regulation, in response to a programme of RT in patients with stable COPD and agematched controls. Furthermore, responses were measured during the RT intervention in a matched cohort of patients with stable COPD who ingested post-exercise protein and carbohydrate supplements. We measured a wider range of candidate genes and proteins than in previous studies and related these responses to RT-induced changes in lean mass and strength.

The study addressed these specific hypotheses:

1. RT-induced increases in lean mass and function are blunted in patients with stable COPD compared with agematched controls.

2. This blunting in patients with COPD is mirrored by increased expression of genes and proteins associated with MPB and inhibition of muscle anabolic signalling.

3. Post-exercise dietary carbohydrate and protein supplementation inhibits muscle catabolic events and augments anabolic signalling, thereby enhancing RT functional effects.

\section{MATERIALS AND METHODS}

Detailed methods are provided in the online supplementary materials and methods.

\section{Subjects}

Patients with stable COPD meeting clinical and spirometric criteria for severe (Global Initiative for Chronic Obstructive Lung Disease (GOLD) stage 3 or 4) COPD (forced expiratory volume in $1 \mathrm{~s}$ $\left(\mathrm{FEV}_{1}\right)$ /forced vital capacity $(\mathrm{FVC})$ ratio $<70 \%, \mathrm{FEV}_{1}<50 \%$ predicted) with significant self-reported exercise limitation (Medical Research Council (MRC) grades 3, 4 or 5) were recruited. Age-matched healthy controls (HCs) were recruited from the local population. Leicestershire Research Ethics Committee provided ethical approval and all participants provided informed written consent. The study was registered with the UK National Research Register (reference N0123192026).

\section{Study design}

Participants undertook maximal intensity voluntary RT for 8 weeks. Patients with COPD received (randomised, double blind) a dietary protein-carbohydrate supplement (COPD (S)) or a non-nutritive placebo (COPD $(\mathrm{P})$ ) at the time of training. HCs received placebo. Outcome assessments and muscle sampling were performed at baseline, $24 \mathrm{~h}$ after the first training session (muscle biopsy and blood insulin only), after 4 weeks and post training (8 weeks). Trial design and patient flow are summarised in online supplementary figure $1 \mathrm{~S}$.

\section{Interventions}

\section{Resistance training}

Participants underwent 8 weeks of maximal voluntary isokinetic lower-limb RT (Cybex II Norm, CSMi, USA). Training was fully supervised and consisted of three sessions per week. Each session comprised five sets of 30 maximal knee extensions at an angular velocity of $180 \%$ s. During training, peak torque $(\mathrm{Nm})$ and total work performed $(\mathrm{J})$ were recorded for each extension.

\section{Nutritional supplementation}

The supplement comprised $19 \mathrm{~g}$ protein and $49 \mathrm{~g}$ glucose polymer carbohydrate (Vitargo Gainers Gold, Swecarb, Sweden) in $500 \mathrm{ml}$ of water. The placebo was an identical volume, noncaloric drink. Supplementation was supervised and took place immediately after each training session. Participants and researchers were blinded to the nutritional intervention. Healthy volunteers received only the placebo.

\section{Outcome measurements}

At baseline, spirometry was measured in the seated position (Model R; Vitalograph, UK) according to accepted standards. ${ }^{22}$ Body mass index was calculated from height and weight.

\section{Muscle biopsies}

Vastus lateralis muscle biopsies were obtained after a fast of at least $4 \mathrm{~h}$ and (apart from baseline samples) $24 \mathrm{~h}$ after the previous training session. Samples were snap frozen, stored in liquid nitrogen and analysed later as described below.

\section{Blood sampling}

Fasted venous blood was collected and analysed for serum insulin concentration (Human Insulin specific RIA kit, Millipore, USA).

\section{Quadriceps muscle function}

Maximum isometric strength was determined during maximal voluntary knee extensor contraction (Cybex II Norm, CSMi, USA). Subsequently, peak torque and total isokinetic work were recorded during two bouts of five maximal repetitions (performed at an angular velocity of $60^{\circ} / \mathrm{s}$ ).

\section{Lean mass}

Whole body and thigh lean mass was measured using dual energy X-ray absorptiometry (Lunar Prodigy Advance, GE Healthcare, UK). Fat-free mass index (FFMI) was calculated from height and body fat free mass. Patients were deemed to have muscle wasting if FFMI $<16 \mathrm{~kg} / \mathrm{m}^{2}$ in men or $<15 \mathrm{~kg} / \mathrm{m}^{2}$ in women. ${ }^{23}$

\section{Muscle biopsy analysis}

A wide range of genes (quantitative reverse transcriptase PCR) and proteins (western blotting with infrared detection) were measured at each time point. Candidate genes and proteins were selected on the basis of known association with the regulation of MPB (20S proteasome, MAFbx, MuRF1 and ZNF216, along with calpain-3), MPS (Akt, p70s6 kinase, GSK3 $\alpha$, GSK3 $\beta$, 4EBP1 and Redd1), myogenesis (MyoD, myogenin and myostatin), transcription (members of the family of Forkhead transcription factors, FOXO1 and FOXO3 and RUNX1) and inflammation (tumour necrosis factor $\alpha$ and interleukin-6 mRNA expression). 


\section{Data analysis}

We estimated from our pulmonary rehabilitation programme that a $20 \%$ increase in strength following training would be clinically and physiologically significant. To detect this strength difference $(80 \%$ power, $\alpha=0.05)$ we required 25 patients to complete training in each group.

We included only subjects who provided at least a baseline and $24 \mathrm{~h}$ time-point muscle biopsy (see online supplementary figure 1S). An independent Student t test was used to compare baseline clinical and functional data between groups. Two-way repeated measures analysis of variance, and when appropriate least significant difference post hoc tests, were used to compare within-group changes over time and differences between treatment groups.

For molecular data, comparison of two independent groups was performed using Mann-Whitney's non-parametric test, and within-group comparison of more than two time points was performed using Friedman's non-parametric analysis. Data in tables and figures are mean \pm SEM. Significance was set at $\mathrm{p}<0.05$.

\section{RESULTS}

Fifty-nine patients with COPD and $21 \mathrm{HCs}$ were included in the analysis. Online supplementary figure $1 \mathrm{~S}$ shows the flow through the study and the number of viable muscle samples at each time point.

\section{Baseline (pretraining)}

Physical characteristics

Baseline characteristics are shown in table 1. There were no differences at baseline between the COPD (P) and COPD (S) groups. Muscle function and whole body exercise performance were lower in patients with COPD than HCs. Thigh lean mass was lower in patients compared with HCs, but this difference

Table 1 Baseline physical characteristics

\begin{tabular}{|c|c|c|c|}
\hline & $\begin{array}{l}\text { Healthy controls } \\
(n=21)\end{array}$ & $\begin{array}{l}\text { COPD (P) } \\
(n=27)\end{array}$ & $\begin{array}{l}\operatorname{COPD}(S) \\
(n=32)\end{array}$ \\
\hline Age (years) & $66.1(1.0)$ & $66.9(1.7)$ & $68.9(1.7)$ \\
\hline Gender (M:F) & 10:11 & $14: 13$ & 19:13 \\
\hline BMI $\left(\mathrm{kg} / \mathrm{m}^{2}\right)$ & $26.8(0.6)$ & $25.5(1.0)$ & $26.6(0.8)$ \\
\hline FFMI $\left(\mathrm{kg} / \mathrm{m}^{2}\right)$ & $17.5(0.3)$ & $16.9(0.4)$ & $17.6(0.4)$ \\
\hline $\begin{array}{l}\text { Subjects with muscle } \\
\text { wastage }(n)\end{array}$ & 0 & 7 & 6 \\
\hline Smoking pack years & $14.4(5.1)^{* * *}$ & $48.1(5.6)$ & $46.9(7.9)$ \\
\hline Physical activity score & $10.3(1.2)^{* * *}$ & $6.1(0.3)$ & $6.8(1.1)$ \\
\hline $\mathrm{FEV}_{1}$ (litres) & $2.5(0.1)^{* * *}$ & $1.1(0.0)$ & $1.12(0.0)$ \\
\hline $\mathrm{FEV}_{1}$ (\% predicted) & $105.0(4.7)^{* * *}$ & $45.8(3.2)$ & $47.7(3.3)$ \\
\hline $\mathrm{FEV}_{1} / \mathrm{FVC}(\%)$ & $71.8(1.4)^{* * *}$ & $41.2(2.1)$ & 39.42.4) \\
\hline Thigh lean mass (g) & 4190 (189) & 3700 (191) & 3829 (153) \\
\hline Isometric peak torque $(\mathrm{Nm})$ & $135.8(9.5)^{* * *}$ & $109.3(9.8)$ & $105.9(7.4)$ \\
\hline Isokinetic peak torque (Nm) & $98.0(7.9)^{* *}$ & $75.8(6.9)$ & $79.9(6.0)$ \\
\hline Isokinetic peak work (J) & $373.8(33.0)^{* *}$ & $284.5(28.2)$ & $307.5(25.2)$ \\
\hline Cycle peak $\mathrm{VO}_{2}(\mathrm{ml} / \mathrm{kg} / \mathrm{min})$ & $22.8(1.4)^{* * *}$ & $14.7(0.9)$ & $14.4(0.6)$ \\
\hline Cycle peak workload (W) & $119.9(8.7)^{* * *}$ & $51.1(4.7)$ & $48.9(3.8)$ \\
\hline
\end{tabular}

Subjects who provided a minimum of a baseline and $24 \mathrm{~h}$ muscle biopsy were included in the analysis (see online supplementary figure 1S). Figures refer to mean (SEM).

Details of the questionnaire used to measure physical activity score is given in the online supplementary materials and methods.

${ }^{*} \mathrm{p} \leq 0.05$; HC versus COPD (S); ${ }^{*} \mathrm{p} \leq 0.05$; HC versus COPD (P).

$\mathrm{BMI}$, body mass index; COPD (P), patients with COPD allocated placebo; COPD (S), patients with COPD allocated supplements; FFMI, fat-free mass index; HC, healthy control. was not statistically significant. Seven COPD (P) and six COPD (S) subjects were deemed to have muscle wasting.

\section{Molecular data}

Baseline protein and mRNA expression levels in patients with COPD as a whole and in HCs are shown in tables 2 and online supplementary table $1 S$ respectively. At baseline, MAFbx and MuRF1 protein expression, phosphorylation of p70s6kinase, Redd1 protein expression, Myogenin and MyoD protein expression and nuclear FOXO1 protein expression were significantly greater in patients with COPD than in HCs.

Baseline myostatin mRNA expression was greater in patients with COPD, but there was no other difference in gene expression between groups.

\section{Training-induced changes}

\section{Functional data}

Thigh lean mass increased from baseline in HCs $(4.1 \%(0.8 \%)$ and $5.4 \%(0.9 \%)$ at 4 and 8 weeks of RT, respectively) and COPD (P) (4.6\% (0.9\%) and 6.2\% (1.7\%)) and COPD (S) $(3.9 \%(1.3 \%)$ and $4.0 \%(1.1 \%))$ groups (figure $1 \mathrm{~A})$. Isometric strength also increased relative to baseline in all groups at 4 and 8 weeks of RT (HC: $10.0 \%$ (4.1\%) and 12.4\% (4.2\%); COPD (P): $16.9 \%$ (4.3\%) and $17.7 \%(3.7 \%)$ COPD (S): $14.6 \%$ $(2.8 \%)$ and $18.0 \%(3.4 \%)$ ) (figure $1 \mathrm{~B}$ ).

There were no differences in training-induced gains in lean mass or strength between the HC group and the COPD (P) and COPD (S) groups. Similarly, the training-induced increase in absolute thigh lean mass was not different between groups (HC: 232 (40) g; COPD (P): 215 (49) g; COPD (S): 148 (43) g).

Table 2 Protein expression measured from muscle biopsies taken at baseline

\begin{tabular}{|c|c|c|}
\hline & Healthy controls $(n=21)$ & COPD $(n=59)$ \\
\hline \multicolumn{3}{|c|}{ Muscle protein breakdown } \\
\hline $20 \mathrm{~S}$ proteasome & $0.61(0.11)$ & $0.74(0.07)$ \\
\hline MAFbx & $0.36(0.05)$ & $0.83(0.12)^{*}$ \\
\hline MuRF1 & $0.30(0.04)$ & $0.69(0.07)^{* *}$ \\
\hline Calpain3 & $0.50(0.08)$ & $0.52(0.04)$ \\
\hline \multicolumn{3}{|c|}{ Muscle protein synthesis } \\
\hline PAkt1/Akt1 & $1.27(0.37)$ & $1.36(0.11)$ \\
\hline PGSK3 $\alpha / G S K 3 \alpha$ & $0.84(0.26)$ & $0.97(0.11)$ \\
\hline PGSK3 $\beta / G S K 3 \beta$ & $1.71(0.38)$ & $1.28(0.19)$ \\
\hline PP70s6k/P70s6k & $0.82(0.10)$ & $1.18(0.10)^{*}$ \\
\hline P4EBP1/4EBP1 & $1.03(0.24)$ & $1.11(0.10)$ \\
\hline Redd1 & $0.50(0.06)$ & $0.85(0.06)^{* *}$ \\
\hline \multicolumn{3}{|l|}{ Myogenesis } \\
\hline Myostatin & $2.70(0.55)$ & $3.18(0.51)$ \\
\hline MyoD & $0.36(0.05)$ & $0.80(0.09)^{* *}$ \\
\hline Myogenin & $0.45(0.06)$ & $0.86(0.11)^{*}$ \\
\hline \multicolumn{3}{|l|}{ Transcription factors } \\
\hline PF0X01/F0X01 & $1.83(0.39)$ & $2.22(0.37)$ \\
\hline PFOX03/FOX03 & $1.42(0.22)$ & $2.16(0.31)$ \\
\hline FOX01 (nuclear) & $0.51(0.04)$ & $0.89(0.07)^{* *}$ \\
\hline FOXO3 (nuclear) & $0.58(0.10)$ & $0.78(0.06)$ \\
\hline \multicolumn{3}{|c|}{$\begin{array}{l}\text { Figures refer to mean (SEM) relative optical density (normalised to either actin or } \\
\text { lamin). } \\
\text { The COPD group refers to the combined placebo and supplement groups at baseline } \\
\text { (prior to intervention). } \\
{ }^{*} p<0.05,{ }^{* *} p<0.01 \text { significantly different from control. } \\
\text { COPD, chronic obstructive pulmonary disease. }\end{array}$} \\
\hline
\end{tabular}



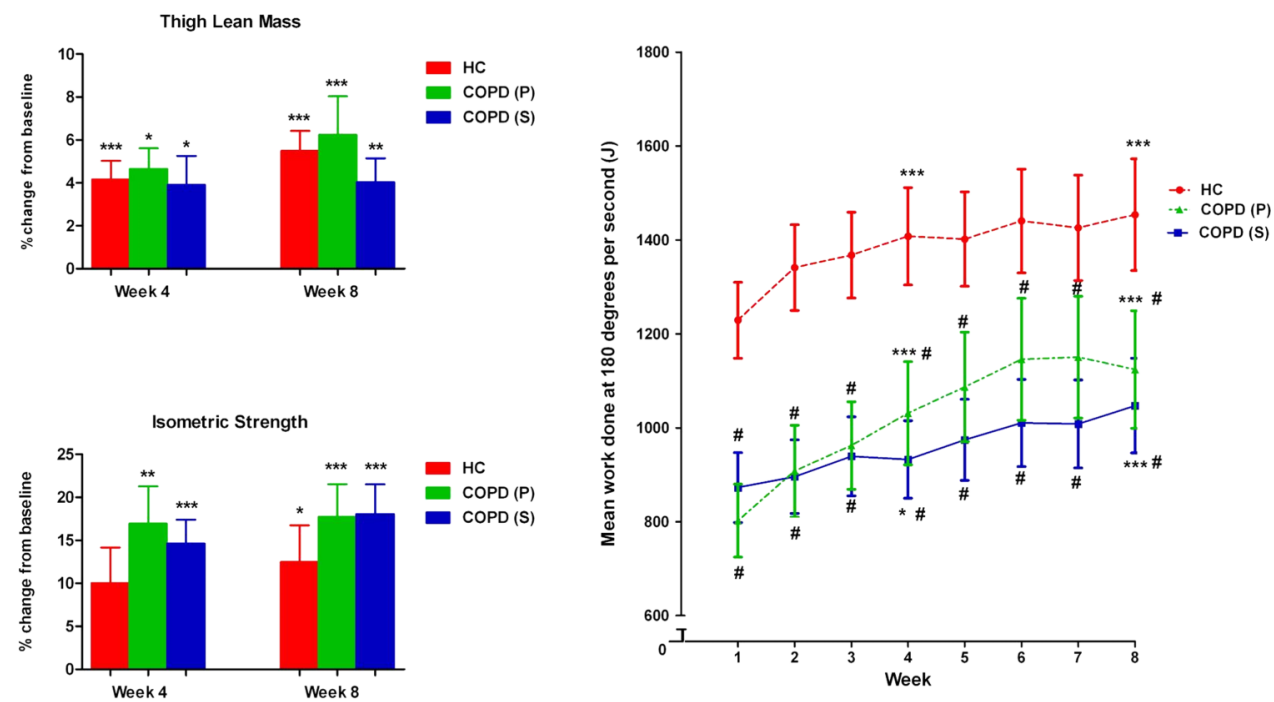

Figure 1 Training-induced changes in muscle mass and strength and progression of work. Changes $(\% \pm S E M)$ from baseline in $(A)$ thigh muscle mass, (B) isometric peak torque in the healthy control group (HC; red bars), chronic obstructive pulmonary disease (COPD) placebo group (COPD (P); green bars) and COPD supplement group (COPD (S); blue bars). ${ }^{*} p<0.05,{ }^{* *} p<0.01,{ }^{* *} p<0.001$ significantly different from baseline. (C) Shows mean $\left( \pm\right.$ SEM) weekly total isokinetic work $\left(180^{\circ} / \mathrm{s}\right)$ performed during the 8 -week training programme in the three groups. ${ }^{\#} p<0.05$ significant difference compared with control group.

Figure 1C shows the weekly progression of total isokinetic work performed during training (weekly average) for each group. Mean absolute work performed by HCs was greater at all stages than in both COPD groups, but there was no difference in the rate of progression of work during training between the $\mathrm{HC}$ and COPD groups and between the COPD (P) and COPD (S) groups.

\section{Training-induced changes in protein expression}

Muscle protein breakdown: In the HC group, expression of proteins involved in MPB was significantly increased $24 \mathrm{~h}$ after the first bout of training and was sustained at 4 and 8 weeks (figure 2A). In patients with COPD, the pattern of change was broadly similar, but of smaller magnitude with fewer changes being statistically significant (figure 2C,D). In particular, the expression of MURF1 and MAFbx was unchanged in both COPD groups during training. There was no difference in the response to training between COPD (P) and COPD (S) groups.

Muscle protein synthesis: In HCs, there was an increase with training in the ratio of phosphorylated protein to total protein expression for all anabolic signalling proteins with the exception of PGSK3 $\beta /$ GSK3 $\beta$ ratio (figure $3 \mathrm{~A}$ ). The pattern of change in COPD was similar, but training-induced changes were of substantially lower magnitude than in HC (figure 3C,D). There was no difference in the magnitude of response when comparing COPD (P) and COPD (S) groups.

Myogenesis: Myostatin protein expression did not change significantly from baseline with training in either HC or COPD groups. There was a statistically significant increase in $\mathrm{MyoD}$ expression after 8 weeks of training in all three groups, which was of the same magnitude. There was a tendency for myogenin protein expression to increase with training in all groups, but this did not reach statistical significance (figure 4A,C,D). There was no difference in the pattern of response to training when comparing COPD (P) and COPD (S) groups.

Transcription factors: Phosphorylated to total protein expression ratios for FOXO1 and FOXO3 transcription factors increased in all groups during training (figure 5). However, the magnitude of change was lower in both COPD groups compared with HCs.

\section{Training-induced changes in mRNA expression}

A description of changes in mRNA expression is provided in the online supplementary materials and methods. Broadly, the pattern change in genes involved in MPB and MPS, myogenesis, transcription and inflammation was similar for HC and COPD (P) and COPD (S) groups (see online supplementary figures 2S-6S). Notably, myostatin mRNA expression was significantly reduced at $24 \mathrm{~h}$, but was restored to the baseline value at 4 and 8 weeks in all groups (see online supplementary figure 4S). Similarly, inflammatory gene expression increased significantly in all groups at $24 \mathrm{~h}$ but was reduced to baseline at 4 and 8 weeks (see online supplementary figure $6 \mathrm{~S}$ ).

\section{DISCUSSION}

This study details the functional and molecular responses of skeletal muscle to RT and post-exercise protein/carbohydrate supplementation combined with RT in patients with COPD and aged-matched HCs. A major finding was that increases in thigh lean mass and knee-extensor strength over 8 weeks of RT in patients with COPD were similar compared with HCs. It is concluded that while baseline muscle function in patients with COPD is compromised, its responsiveness to RT is preserved.

RT increased anabolic, catabolic and transcription factor protein expression (not unexpected given exercise increases muscle protein turnover), but the magnitude of increase was blunted in patients with COPD. This was surprising given that thigh lean mass and strength gains were similar, and suggests a disconnection between changes in protein expression and lean mass gains. There appeared to be a closer association between anabolic, catabolic and transcription factor protein expression levels and work done during RT as the latter was consistently lower in patients with COPD (figure 1C). However, changes in myogenic protein expression with RT were similar in patients with COPD and HCs and may explain the similarity in lean mass gains. This is in line with the observation that testosterone- 
A
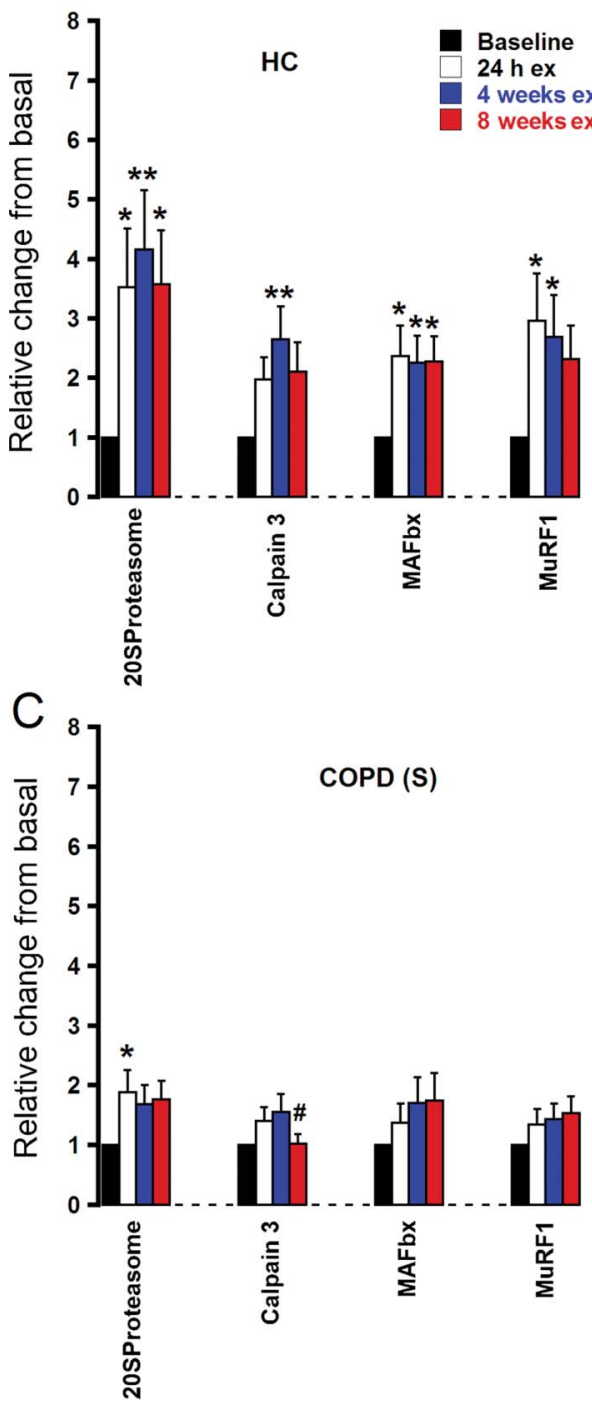

B
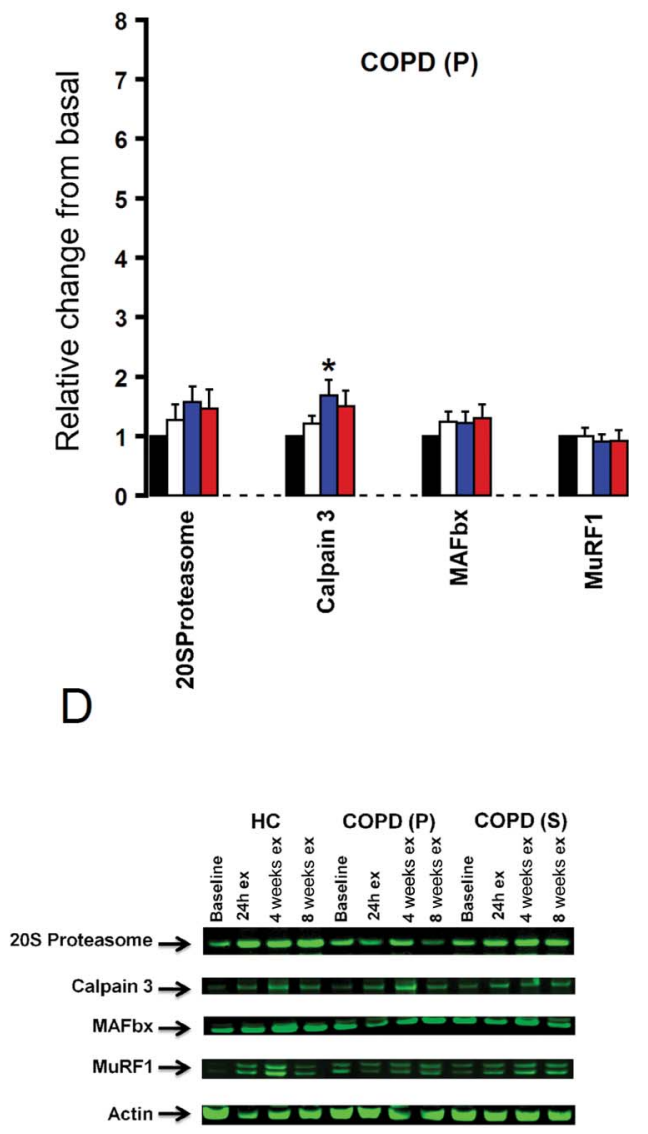

Figure 2 Expression of target proteins regulating muscle protein breakdown in response to training. Protein expression (mean $\pm \mathrm{SEM}$ ) is represented as relative changes from basal and is quantified by western blotting. (A) HC, healthy control group; (B) COPD (P), patients with chronic obstructive pulmonary disease (COPD) receiving placebo; (C) COPD (S), patients with COPD receiving supplement; (D) typical western blot using IRdye 800 (green) secondary antibodies to quantify the catabolic proteins studied. ${ }^{*} \mathrm{p}<0.05,{ }^{* *} \mathrm{p}<0.01$ significantly different from baseline; ${ }^{*} \mathrm{p}<0.05$ significantly different from $24 \mathrm{~h}$. ex, exercise.

mediated muscle hypertrophy in older people is associated with increased myogenin protein expression and satellite cell activation. ${ }^{24}$ Contrary to our hypothesis, post-exercise dietary supplementation in patients with COPD did not alter target gene and protein expression or leg lean mass and functional gains compared with training alone.

Single time-point studies comparing muscle anabolic and catabolic mRNA and protein expression in patients with COPD and HCs have proposed muscle atrophy in COPD occurs as a consequence of increased ubiquitin proteosome mediated $\mathrm{MPB}$, and that increased anabolic signalling may occur as a compensatory phenomenon. ${ }^{1113}$ Conversely, others report little differences in anabolic gene and protein expression levels between patients with COPD and HCs. ${ }^{12}$ In this study MuRF1, MAFbx and nuclear FOXO1 protein expression was greater in patients with COPD than in HCs at baseline (table 2). However, these traits were present even when thigh lean tissue mass and muscle inflammatory cytokine mRNA expression were similar between patients with COPD and HCs (table 2, see online supplementary table 1S), suggesting these differences may be features of deconditioning rather than increased MPB and wasting per se, which is supported by the lack of difference in proteasome protein expression at baseline. In the absence of MPB measurements it is not possible to be more conclusive but increased muscle FOXO and MAFbx protein expression has been reported under conditions of altered muscle carbohydrate and lipid oxidation and in the absence of muscle wasting. ${ }^{25}$ The greater muscle catabolic protein expression at baseline in patients with COPD was also paralleled by greater expression of selected anabolic and myogenic signalling proteins, perhaps suggesting greater basal muscle protein turnover in COPD.

To our knowledge, no COPD study has documented the time course of muscle molecular events under conditions when muscle mass has been increased by RT. Muscle cytokine mRNA expression increased transiently in response to training, but the magnitude was the same in patients with COPD and HCs (see 
A
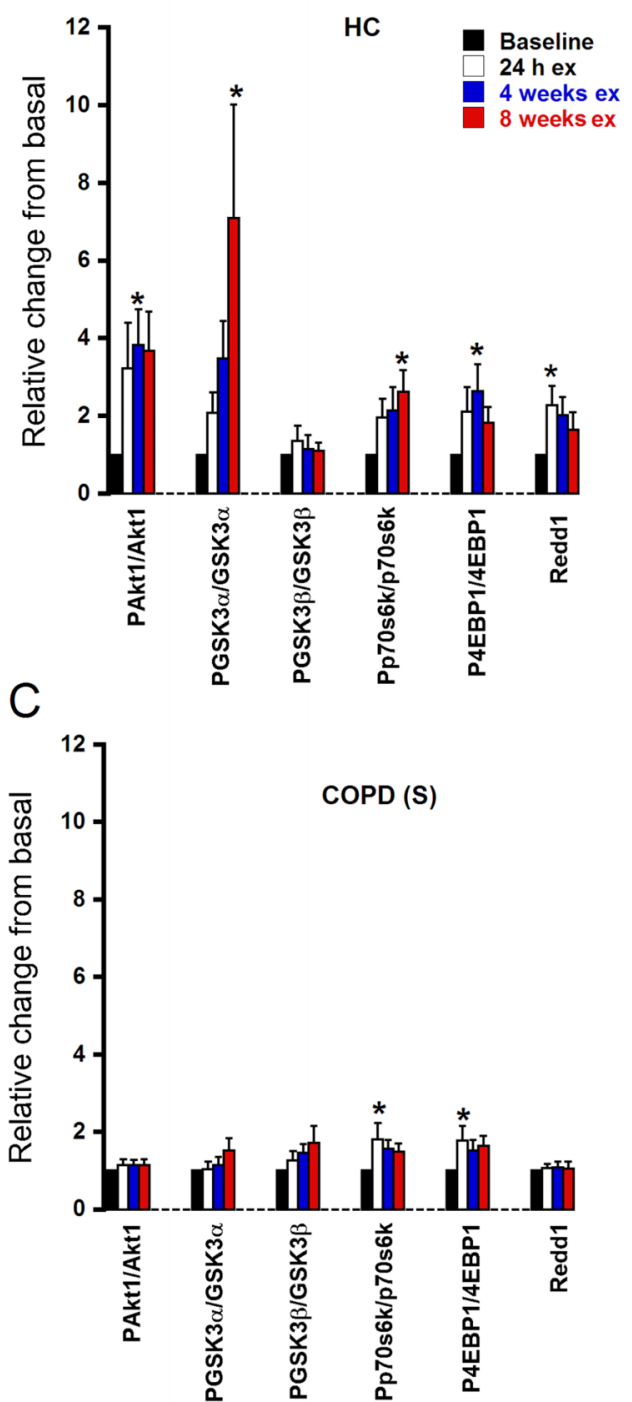

B

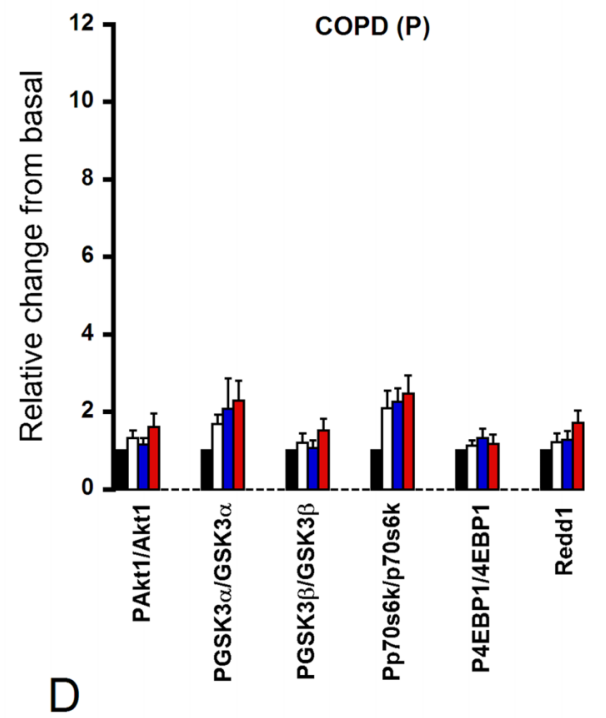

Figure 3 Expression of target proteins regulating muscle protein synthesis in response to training. Protein expression (mean $\pm S E M)$ is represented as relative changes from basal and is quantified by western blotting. (A) HC, healthy control group; (B) COPD (P), patients with chronic obstructive pulmonary disease (COPD) receiving placebo; (C) COPD (S), patients with COPD receiving supplement; (D) typical western blot using IRDye 800 (green) and IRDye 680 (red) secondary antibodies to quantify the anabolic proteins studied. ${ }^{*} \mathrm{p}<0.05$ significantly different from baseline. ex, exercise.

online supplementary figure 6S), and likely reflected an acute inflammatory response to unaccustomed exercise. Furthermore, this response, together with the transitory decrease in myostatin mRNA expression over the same time course, demonstrates short-term changes in mRNA abundance may be of limited physiological relevance in the absence of associated protein changes. This point is further substantiated by the lack of close alliance between changes in mRNA and protein abundance with training for each molecular target in this study. The current study extends previous reports (which generally focused on relatively few mRNA and protein targets ${ }^{26}$ ) by documenting responses of a substantially wider range of targets to a training intervention that increased lean tissue mass and strength, capturing time-course changes. The disconnection between protein expression levels and muscle mass gains with RT is supported by data showing that increasing amino acid and insulin availability (thus doubling leg protein synthesis and halving leg protein breakdown in young, healthy volunteers) did not result in parallel changes in anabolic signalling activity (phosphorylation of the Akt/mTOR/P70S6k/eIF4F pathway), and more simply reflected changes in insulin availability. ${ }^{17}$ Additionally, the decline in MPS observed during limb immobilisation in healthy, young volunteers is not reflected by changes in expression levels of mTOR signalling proteins, which remained unchanged from basal. ${ }^{27}$ It could be argued that measuring anabolic, catabolic and transcription factor protein expression in the resting fasted state (as in the present study) does not provide significant insight regarding molecular responses mediated at the time of exercise, but if this is the case it is difficult to reconcile the changes in mRNA and protein expression levels we observed with RT in the present study. A likely explanation is that closer association exists between changes in protein expression levels and work done during training than lean mass gains. In support of this, Burd et $a l^{28}$ recently demonstrated that the volume (not intensity) of work done during an acute bout of resistance exercise is positively associated with the magnitude of post-exercise 
A

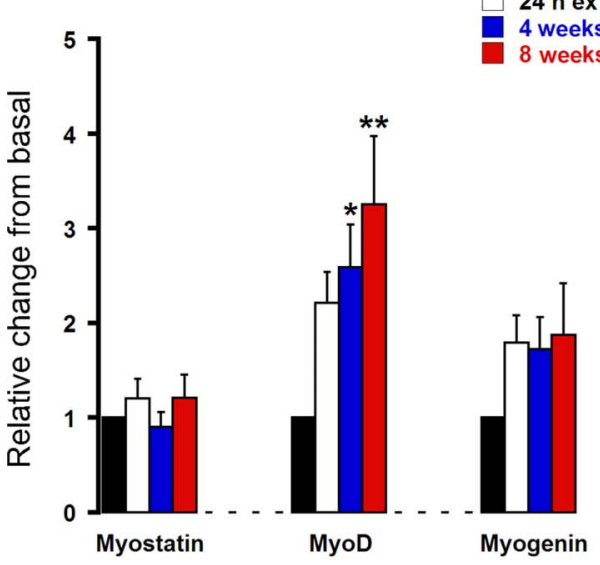

COPD (S)



B

$\operatorname{COPD}(\mathrm{P})$


Figure 4 Expression of target proteins regulating myogenesis in response to training. Protein expression (mean \pm SEM) is represented as relative changes from basal and is quantified by western blotting. (A) HC, healthy control group; (B) COPD (P), patients with chronic obstructive pulmonary disease (COPD) receiving placebo; (C) COPD (S), patients with COPD receiving supplement; (D) typical western blot using IRDye 800 (green) secondary antibodies to quantify the myogenic proteins studied. ${ }^{*} p<0.05,{ }^{* *} p<0.01$ significantly different from baseline; ${ }^{\#} p<0.05$ significantly different from $24 \mathrm{~h}$. ex, exercise.

p7056K phosphorylation, and that this relationship exists for up to $30 \mathrm{~h}$ following exercise which is considerably longer than in the present study. Furthermore recent work shows the intensity of chronic RT does not determine the magnitude of training-induced muscle hypertrophy in young men. ${ }^{29}$ As this study demonstrates, this has important implications for muscle rehabilitation in COPD and other wasting diseases.

Anabolic resistance of muscle to protein nutrition is a feature of ageing and has been proposed to be a causative factor in sarcopenia. ${ }^{1920}$ It has been suggested that dietary supplementation might need to be combined with muscle contraction to facilitate muscle protein accretion in ageing. ${ }^{19}{ }^{30}$ Despite supplementation occurring immediately after each bout of training when the anabolic response to feeding is maximised, ${ }^{21}$ we did not observe an effect of feeding on molecular or functional outcomes, suggesting dietary protein intake is not a major limitation to RT-induced muscle mass gains in COPD.

Controversy exists about whether impaired muscle mass and function can be ascribed to COPD-specific factors (eg, systemic inflammation, hypoxia, drug therapy) or is predominantly due to physical inactivity. Our observation that RT increased lean mass and strength in COPD, and by the same magnitude as observed in HCs, suggests inactivity and deconditioning are key factors underpinning muscle dysfunction in COPD, although we recognise that muscle dysfunction due to disease-specific factors may also be modifiable by training. Importantly, muscle mass and function increased with RT even in the face of increased catabolic mRNA and protein expression, which probably reflects exercise-induced increases in muscle protein turnover. We recognise our observations are limited to patients with stable disease and preserved muscle mass who were able to undertake a demanding RT programme with repeated muscle biopsy sampling. However, we selected patients with significant selfreported exercise limitation (MRC grades 3-5), demonstrating markedly impaired muscle strength and aerobic capacity at baseline, suggesting the population represented those referred for pulmonary rehabilitation.

In conclusion, we demonstrated there is no disease-specific barrier to increasing lean tissue mass and function through RT in patients with COPD and lung impairment, exercise intolerance and weakness at baseline. We also showed that increasing post-exercise dietary protein and carbohydrate intake is not a prerequisite for a normal training response in COPD. Our observation that (with the exception of myogenic proteins) 
A

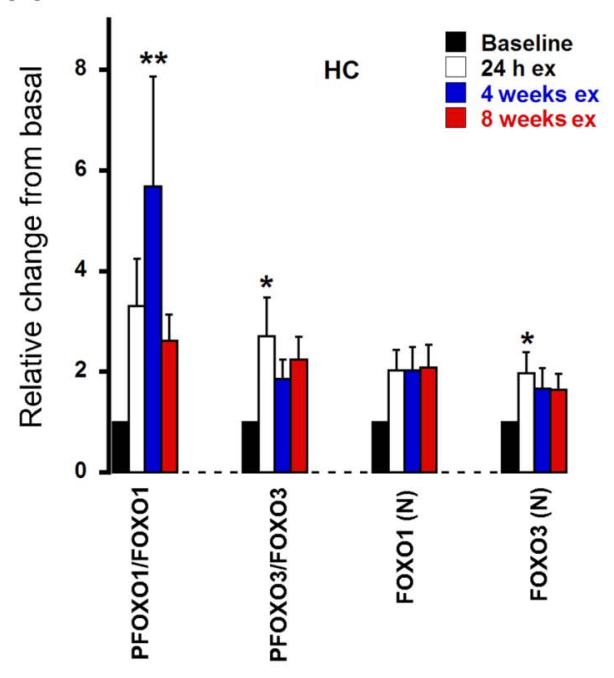

C

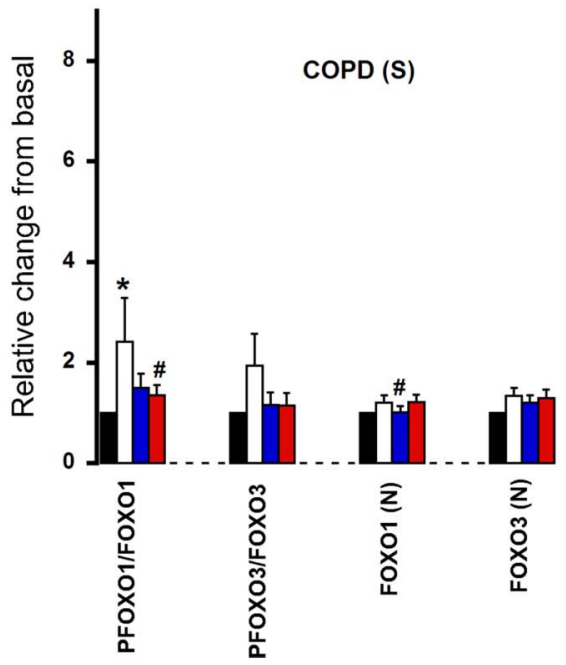

B

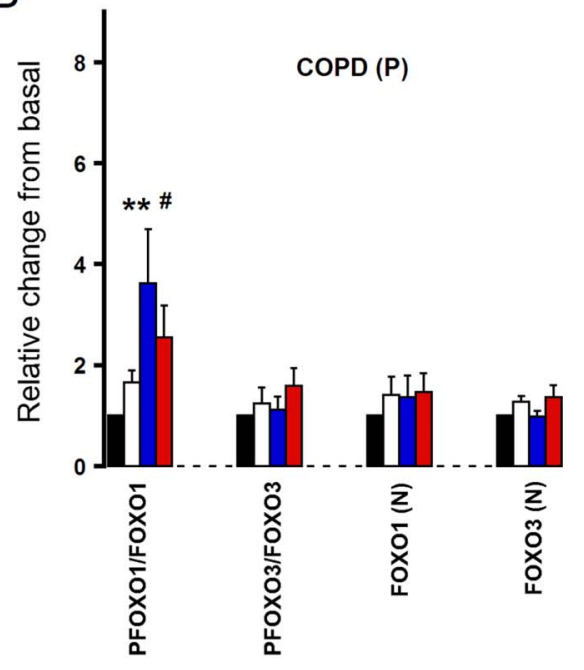

D

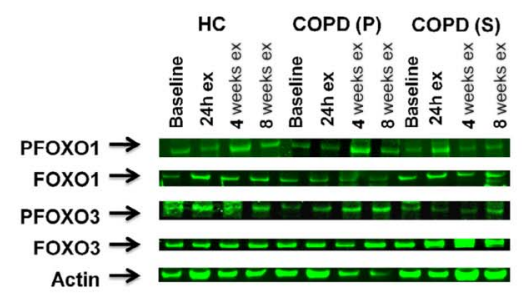

Figure 5 Expression of target transcription factor proteins in response to training. Protein expression (mean \pm SEM) is represented as relative changes from basal and is quantified by western blotting. (A) HC, healthy control group; (B) COPD (P), patients with chronic obstructive pulmonary disease (COPD) receiving placebo; (C) COPD (S), patients with COPD receiving supplement; (D) typical western blot using IRDye 800 (green) secondary antibodies to quantify the transcription factors studied. ${ }^{*} p<0.05,{ }^{*} p<0.01$ significantly different from baseline; ${ }^{\#} p<0.05$ significantly different from 24 h. ex, exercise; (N), nuclear.

gains in lean mass are not tightly coupled to the magnitude of change in protein expression suggests some caution when identifying potential targets for intervention.

Acknowledgements This study was supported by the Medical Research Council (grant award G0501985). We would like to thank Sally Cordon for performing the insulin assay and Caroline Sandland and Samantha Harrison for assistance with supervising the resistance training. We would like to thank Swecarb (Sweden) for providing the dietary supplements.

Contributors DC conducted wet laboratory procedures and molecular data analysis. MKM recruited subjects, conducted outcome assessments (including muscle biopsies) and data analysis. LH-W supervised resistance training and nutritional supplementation and contributed to data analysis. MDM and SJS helped plan and coordinate the study and supervised staff working on the project. PG and MCS wrote the original grant application, developed the study protocol, supervised staff working on the project and contributed to data analysis. All authors contributed to manuscript writing.

Funding UK Medical Research Council (grant G0501985).

Competing interests None.
Ethics approval Leicestershire Research Ethics Committee.

Provenance and peer review Not commissioned; externally peer reviewed.

\section{REFERENCES}

1 American Thoracic Society. Skeletal muscle dysfunction in chronic obstructive pulmonary disease. A statement of the American Thoracic Society and European Respiratory Society. Am J Respir Crit Care Med 1999;159(4 Pt 2):S1-40.

2 Fabbri LM, Rabe KF. From COPD to chronic systemic inflammatory syndrome? Lancet 2007;370:797-9.

3 Agusti A. Systemic effects of chronic obstructive pulmonary disease: what we know and what we don't know (but should). Proc Am Thorac Soc 2007;4:522-5.

4 Marquis K, Debigare R, Lacasse $Y$, et al. Midthigh muscle cross-sectional area is a better predictor of mortality than body mass index in patients with chronic obstructive pulmonary disease. Am J Respir Crit Care Med 2002;166:809-13.

5 Swallow EB, Reyes D, Hopkinson NS, et al. Quadriceps strength predicts mortality in patients with moderate to severe chronic obstructive pulmonary disease. Thorax 2007;62:115-20.

6 Schols AM, Mostert R, Soeters PB, et al. Nutritional state and exercise performance in patients with chronic obstructive lung disease. Thorax 1989;44:937-41.

7 Vestbo J, Prescott E, Almdal T, et al. Body mass, fat-free body mass, and prognosis in patients with chronic obstructive pulmonary disease from a random population 
sample: findings from the Copenhagen City Heart Study. Am J Respir Crit Care Med 2006;173:79-83.

8 Steiner MC, Roubenoff $\mathrm{R}$, Tal-Singer $\mathrm{R}$, et al. Prospects for the development of effective pharmacotherapy targeted at the skeletal muscles in chronic obstructive pulmonary disease: a translational review. Thorax 2012:67:1102-9.

9 Bernard S, Whittom F, LeBlanc $P$, et al. Aerobic and strength training in patients with chronic obstructive pulmonary disease. Am I Respir Crit Care Med 1999;159:896-901.

10 Kongsgaard $\mathrm{M}$, Backer $\mathrm{V}$, Jorgensen $\mathrm{K}$, et al. Heavy resistance training increases muscle size, strength and physical function in elderly male COPD-patients-a pilot study. Respir Med 2004;98:1000-7.

11 Doucet M, Russell AP, Leger B, et al. Muscle atrophy and hypertrophy signaling in patients with chronic obstructive pulmonary disease. Am I Respir Crit Care Med 2007:176:261-9.

12 Plant PJ, Brooks D, Faughnan M, et al. Cellular markers of muscle atrophy in chronic obstructive pulmonary disease. Am J Respir Cell Mol Biol 2010;42:461-71.

13 Debigare $\mathrm{R}$, Maltais $\mathrm{F}$, Cote $\mathrm{CH}$, et al. Profiling of mRNA expression in quadriceps of patients with COPD and muscle wasting. COPD 2008;5:75-84.

14 Rennie MJ, Wackerhage $\mathrm{H}$, Spangenburg EE, et al. Control of the size of the human muscle mass. Annu Rev Physiol 2004;66:799-828.

15 Hulmi JJ, Kovanen V, Selanne $\mathrm{H}$, et al. Acute and long-term effects of resistance exercise with or without protein ingestion on muscle hypertrophy and gene expression. Amino Acids 2009:37:297-308.

16 Campbell WW, Leidy HJ. Dietary protein and resistance training effects on muscle and body composition in older persons. J Am Coll Nutr 2007:26:696S-703S.

17 Greenhaff PL, Karagounis LG, Peirce N, et al. Disassociation between the effects of amino acids and insulin on signaling, ubiquitin ligases, and protein turnover in human muscle. Am J Physiol Endocrinol Metab 2008;295:E595-604.

18 Wilkes EA, Selby AL, Atherton PJ, et al. Blunting of insulin inhibition of proteolysis in legs of older subjects may contribute to age-related sarcopenia. Am J Clin Nutr 2009;90:1343-50.

19 Cuthbertson D, Smith K, Babraj J, et al. Anabolic signaling deficits underlie amino acid resistance of wasting, aging muscle. FASEB J 2005;19:422-4.
20 Kumar V, Selby A, Rankin D, et al. Age-related differences in the dose-response relationship of muscle protein synthesis to resistance exercise in young and old men. J Physiol 2009:587(Pt 1):211-17.

21 Esmarck B, Andersen JL, Olsen S, et al. Timing of postexercise protein intake is important for muscle hypertrophy with resistance training in elderly humans. J Physiol 2001;535(Pt 1):301-11.

22 Quanjer PH, Tammeling GJ, Cotes JE, et al. Lung volumes and forced ventilatory flows. Report Working Party Standardization of Lung Function Tests, European Community for Steel and Coal. Official Statement of the European Respiratory Society. Eur Respir J Supp/ 1993;16:5-40.

23 Schols AM, Broekhuizen R, Weling-Scheepers CA, et al. Body composition and mortality in chronic obstructive pulmonary disease. Am J Clin Nutr 2005;82:53-9.

24 Sinha-Hikim I, Cornford M, Gaytan H, et al. Effects of testosterone supplementation on skeletal muscle fiber hypertrophy and satellite cells in community-dwelling older men. J Clin Endocrinol Metab 2006;91:3024-33.

25 Constantin D, McCullough J, Mahajan RP, et al. Novel events in the molecular regulation of muscle mass in critically ill patients. J Physiol 2011;589(Pt 15):3883-95.

26 Troosters T, Probst VS, Crul T, et al. Resistance training prevents deterioration in quadriceps muscle function during acute exacerbations of chronic obstructive pulmonary disease. Am J Respir Crit Care Med 2010;181:1072-7.

27 de Boer MD, Selby A, Atherton $\mathrm{P}$, et al. The temporal responses of protein synthesis, gene expression and cell signalling in human quadriceps muscle and patellar tendon to disuse. J Physiol 2007:585(Pt 1):241-51.

28 Burd NA, Andrews RJ, West DW, et al. Muscle time under tension during resistance exercise stimulates differential muscle protein sub-fractional synthetic responses in men. J Physiol 2012;590(Pt 2):351-62.

29 Mitchell CJ, Churchward-Venne TA, West DW, et al. Resistance exercise load does not determine training-mediated hypertrophic gains in young men. J Appl Physiol 2012;113:71-7

30 Dorrens J, Rennie MJ. Effects of ageing and human whole body and muscle protein turnover. Scand J Med Sci Sports 2003;13:26-33. 